\title{
Article \\ Overlapping Functions of the Paralogous Proteins AtPAP2 and AtPAP9 in Arabidopsis thaliana
}

\author{
Renshan Zhang ${ }^{1}{ }^{\circledR}$, Xiaoqian Guan ${ }^{1}$, Meijing Yang ${ }^{1}$, Yee-Song Law ${ }^{1}{ }^{\circ}$, Chia Pao Voon ${ }^{1}$, Junran Yan ${ }^{1}$, Feng Sun ${ }^{1}$ \\ and Boon Leong $\operatorname{Lim}^{1,2, * \mathbb{D}}$ \\ 1 School of Biological Sciences, The University of Hong Kong, Hong Kong, China; \\ rs_zhang@fudan.edu.cn (R.Z.); sophia.xiaoqianguan@gmail.com (X.G.); celine30@connect.hku.hk (M.Y.); \\ yeesong0210@gmail.com (Y.-S.L.); VoonChiaPao@hotmail.com (C.P.V.); judithayan@gmail.com (J.Y.); \\ epusun@sdu.edu.cn (F.S.) \\ 2 State Key Laboratory of Agrobiotechnology, The Chinese University of Hong Kong, Hong Kong, China \\ * Correspondence: bllim@hku.hk; Tel.: +852-2299-0826; Fax: +852-2559-9114
}

Citation: Zhang, R.; Guan, X.; Yang, M.; Law, Y.-S.; Voon, C.P.; Yan, J.; Sun, F.; Lim, B.L. Overlapping Functions of the Paralogous Proteins AtPAP2 and AtPAP9 in Arabidopsis thaliana. Int. J. Mol. Sci. 2021, 22, 7243. https:// doi.org/10.3390/ijms22147243

Academic Editors: Bartolome Sabater Elke Barbez and Sascha Waidmann

Received: 14 May 2021

Accepted: 1 July 2021

Published: 6 July 2021

Publisher's Note: MDPI stays neutral with regard to jurisdictional claims in published maps and institutional affiliations.

Copyright: (c) 2021 by the authors. Licensee MDPI, Basel, Switzerland. This article is an open access article distributed under the terms and conditions of the Creative Commons Attribution (CC BY) license (https:// creativecommons.org/licenses/by/ $4.0 /)$.

\begin{abstract}
Arabidopsis thaliana purple acid phosphatase 2 (AtPAP2), which is anchored to the outer membranes of chloroplasts and mitochondria, affects carbon metabolism by modulating the import of some preproteins into chloroplasts and mitochondria. AtPAP9 bears a $72 \%$ amino acid sequence identity with AtPAP2, and both proteins carry a hydrophobic motif at their C-termini. Here, we show that AtPAP9 is a tail-anchored protein targeted to the outer membrane of chloroplasts. Yeast two-hybrid and bimolecular fluorescence complementation experiments demonstrated that both AtPAP9 and AtPAP2 bind to a small subunit of rubisco 1B (AtSSU1B) and a number of chloroplast proteins. Chloroplast import assays using [ $\left[{ }^{35} \mathrm{~S}\right]$-labeled AtSSU1B showed that like AtPAP2, AtPAP9 also plays a role in AtSSU1B import into chloroplasts. Based on these data, we propose that AtPAP9 and AtPAP2 perform overlapping roles in modulating the import of specific proteins into chloroplasts. Most plant genomes contain only one PAP-like sequence encoding a protein with a hydrophobic motif at the C-terminus. The presence of both AtPAP2 and AtPAP9 in the Arabidopsis genome may have arisen from genome duplication in Brassicaceae. Unlike AtPAP2 overexpression lines, the AtPAP9 overexpression lines did not exhibit early-bolting or high-seed-yield phenotypes. Their differential growth phenotypes could be due to the inability of AtPAP9 to be targeted to mitochondria, as the overexpression of AtPAP2 on mitochondria enhances the capacity of mitochondria to consume reducing equivalents.
\end{abstract}

Keywords: AtPAP2; AtPAP9; chloroplasts; import; mitochondria; purple acid phosphatase

\section{Introduction}

Purple acid phosphatases (PAPs) are present in yeasts, animals, and plants. These enzymes contain seven conserved metal-coordinating amino acid residues in five invariant blocks-DXG, GDXXY, GNH(D/E), VXXH, and GHXH [1,2]. The functional roles of plant purple acid phosphatases are diverse, including mobilization of internal and external phosphorus [3,4], cell wall generation [5], use of extracellular ATP [6], and regulation of stress responses [7]. Among the 29 purple acid phosphatases (AtPAPs) in the Arabidopsis genome, only AtPAP2 and AtPAP9 carry an extended hydrophobic C-terminal motif at their C-termini. AtPAP2 is targeted to the outer membranes of both plastids and mitochondria by this unique C-terminal hydrophobic motif [8,9] and plays a role in the import of some nucleus-encoded proteins into chloroplasts and mitochondria [10,11]. Many nucleus-encoded proteins imported into chloroplasts and mitochondria are targeted by their N-terminal transit peptides/presequences, which are recognized by receptor proteins in the translocon of the outer membranes of chloroplasts (TOC) and translocon of the outer membranes of mitochondria (TOM), respectively $[12,13]$. Some transit 
peptides / presequences are phosphorylated by cytosolic STY kinases [14,15], and the dephosphorylation of these phosphorylated transit peptides/presequences is required prior to the import of proteins into chloroplasts and mitochondria [16,17]. AtPAP2 is a phosphatase on the outer membranes of chloroplasts and mitochondria that recognizes these phosphorylated preproteins $[10,11]$. The overexpression of AtPAP2 on both organelles promotes the plant growth and seed yield of Arabidopsis thaliana by coordinating the physiology of chloroplasts and mitochondria simultaneously $[8,18]$. The chloroplasts of the AtPAP2 OE line exhibit a higher PSI/PSII ratio and ETR and fix more $\mathrm{CO}_{2}$, whereas the mitochondria of the AtPAP2 OE line can consume more reducing equivalents and generate more ATP compared with the WT line [19]. The higher consumption of reducing equivalents in the mitochondria can indirectly enhance the regeneration of stromal $\mathrm{NADP}^{+}$, electron acceptors of the linear electron flow [19], and thus the leaves of the AtPAP2 overexpression line contain higher ATP/NADPH and $\mathrm{NADP}^{+} / \mathrm{NADPH}$ ratios during photosynthesis compared with the WT line [18]. Surprisingly, when AtPAP2 is solely overexpressed in mitochondria, the transgenic lines exhibit high leaf-ATP, low sucrose, low seed-yield, and early-senescence phenotypes, possibly due to overreactive mitochondria [20].

AtPAP9 displays a $72 \%$ sequence similarity with AtPAP2, and both phosphatases carry hydrophobic C-terminal motifs $[8,21]$. Here, we show that AtPAP9 and AtPAP2 serve partially redundant biological functions in chloroplast protein import. However, unlike AtPAP2, overexpression of AtPAP9 in Arabidopsis thaliana does not promote plant growth, possibly due to its inability to be targeted to mitochondria and modulate mitochondrial activity [19].

\section{Results}

\subsection{The C-Terminal Hydrophobic Motif of AtPAP9 Targets GFP to Chloroplasts}

Previous studies have shown that AtPAP2 is dually targeted to plastids and mitochondria via its C-terminal hydrophobic motif $[8,22]$. The C-terminal hydrophobic motif of AtPAP9 (Figure S1) has a hydrophobicity value that is similar to that of AtPAP2 as well as that of the translocase of the outer chloroplast membrane (TOC) and the translocase of the outer mitochondrial membrane (TOM) (Table S1) [8]. Given the high sequence similarity (72\% amino acid identity) between AtPAP2 and AtPAP9 (Figure S2), we investigated the targeting ability of the AtPAP9 C-terminal hydrophobic motif with green fluorescence protein (GFP) tagging. Forty-six amino acid residues at the C-terminus of AtPAP9 (a.a. 606-651) were fused to the C-terminus of GFP, and transient expression of the GFP fusion constructs was performed in Arabidopsis protoplasts.

Fluorescence microscopy analysis revealed that the GFP fused to the C-terminal hydrophobic motif of AtPAP9 (GFP-P9C) is targeted to the outer membrane of chloroplasts but does not co-localize with the mitochondrial marker. RFP fused with the mitochondrial ATP synthase subunit beta 1 (F1-RFP), whereas the C-terminal hydrophobic motif of AtPAP2 (GFP-P2C1) was targeted to the outer membranes of both chloroplasts and mitochondria (Figure 1). This indicated that the C-terminal hydrophobic motif of AtPAP9 targets GFP to chloroplasts but not to mitochondria. Although both C-terminal hydrophobic motifs have similar hydrophobicity (Table S1), physio-chemical properties, such as the net charge of the franking amino acids, may also affect targeting [23]. A comparison of the transmembrane helix motifs of AtPAP2 and AtPAP9 (orange box in Figure S2) showed a negatively charged glutamate (E) at position a.a. 609 in AtPAP9, but this position (a.a. 620 in AtPAP2) was a positively charged lysine (K) in AtPAP2 (Figure S2). In addition, nine amino acids upstream of the C-terminal motif in AtPAP2 were missing in AtPAP9 (Figure S2). All of these could affect the targeting of AtPAP9 to mitochondria. 


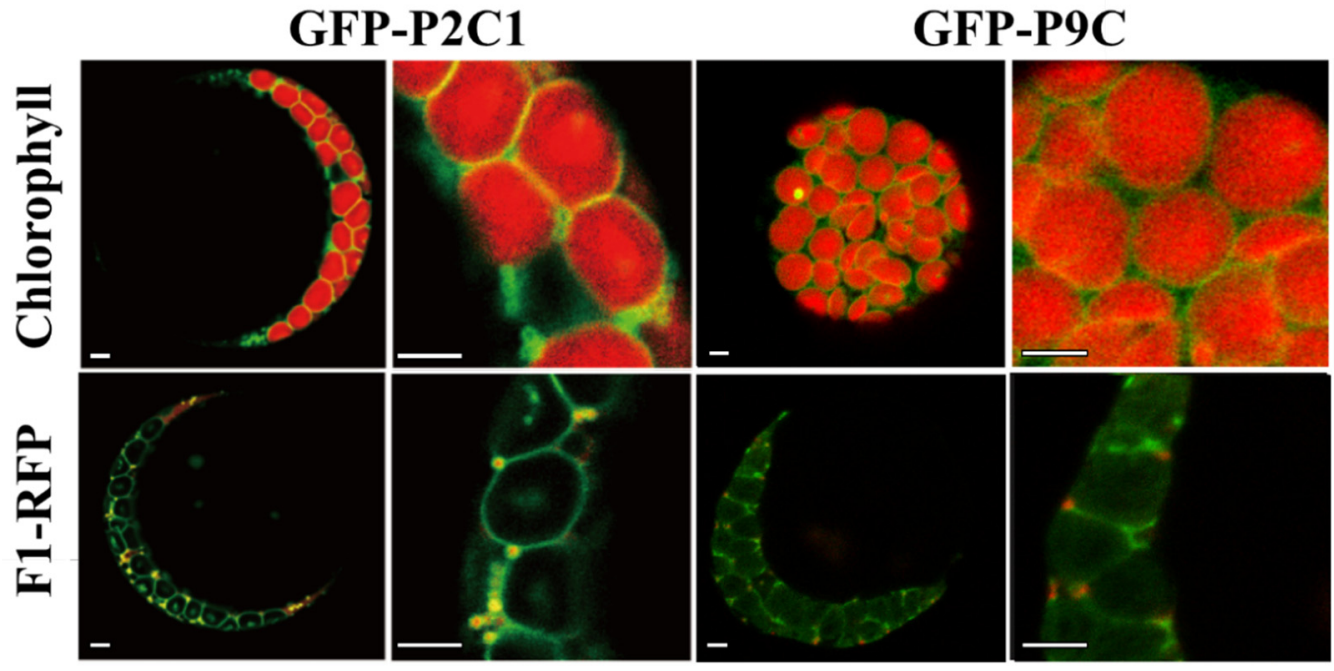

Figure 1. Targeting of GFP to organelles in Arabidopsis protoplasts by the C-terminal motifs of AtPAP2 and AtPAP9. GFP-P2C1 was targeted to the outer membranes of chloroplasts and mitochondria, and GFP-P9C was targeted to the outer membrane of only chloroplasts. Chlorophyll was the chloroplast marker, and F1-RFP was the mitochondrion marker. Scale bar $=5 \mu \mathrm{m}$.

\subsection{Overexpression Lines of AtPAP9 Did Not Exhibit Growth-Promoting Phenotypes}

To decipher the role of AtPAP9 in vivo, we generated $\mathrm{T}_{3}$ homozygous AtPAP9 overexpression (OE) lines driven by the cauliflower mosaic virus (CaMV) 35S promoter. Two homozygous lines expressing full-length AtPAP9, P9OE5, and P9OE10 (Figure 2A) were selected from multiple independent lines with high AtPAP9 protein expression (Figure 2B). A homozygous T-DNA line of AtPAP9 (Salk_129905) was obtained from the Arabidopsis Biological Resource Center (ABRC). The T-DNA insertion at the first exon of AtPAP9 (+465 nucleotide from the ATG start codon) abolished its protein expression (Figure 2B).

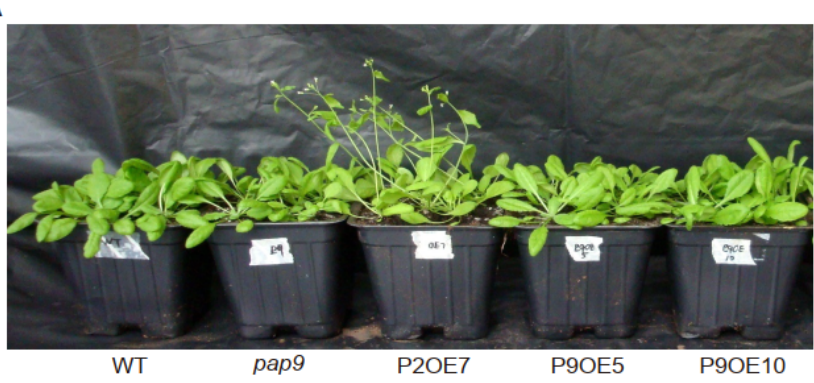

B

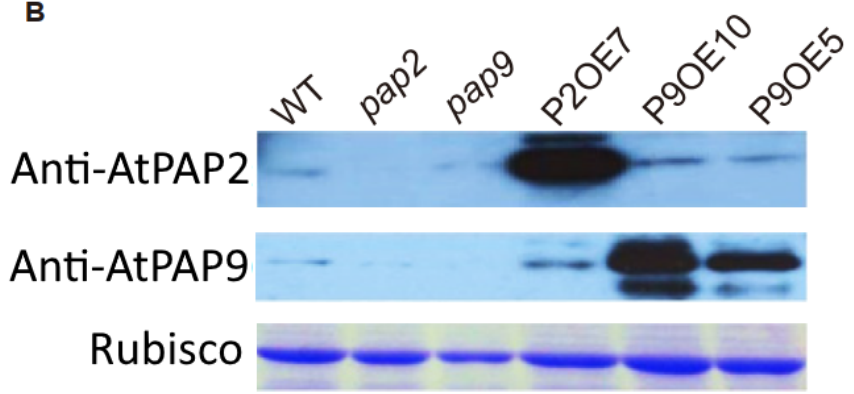

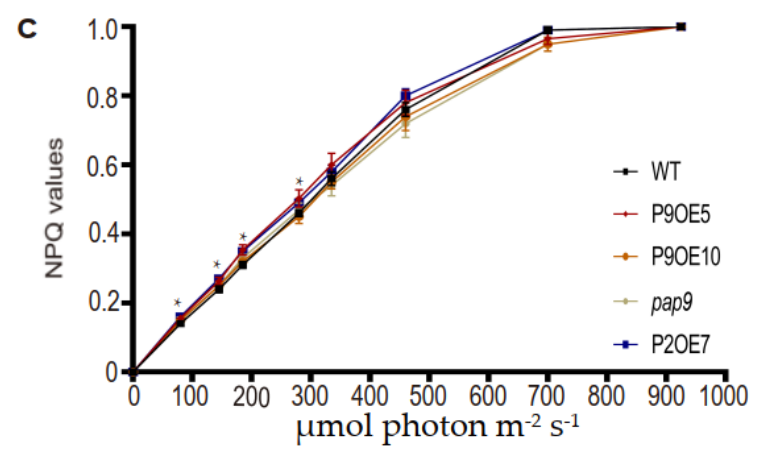

D

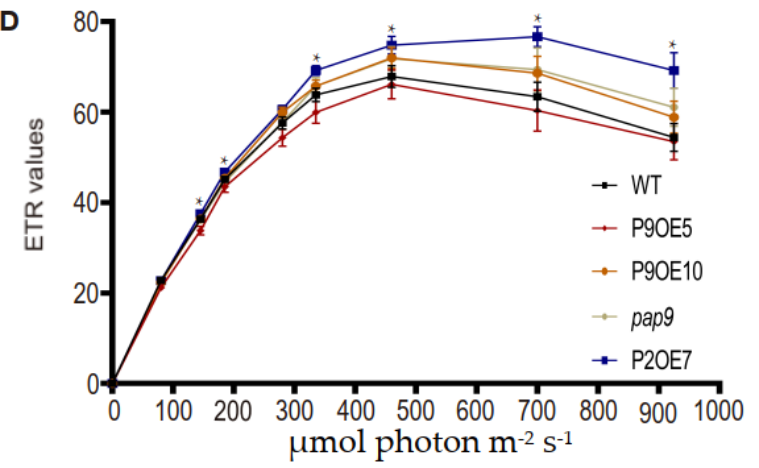

Figure 2. Growth phenotypes of AtPAP9 OE lines. (A) Thirty-day-old soil-sown transgenic plants growing under long-day 
conditions $\left(16 \mathrm{~h}\right.$ light at $22{ }^{\circ} \mathrm{C} / 8 \mathrm{~h}$ dark at $\left.18{ }^{\circ} \mathrm{C}\right)$. Each black soil pot contained nine individual plants of the same line. Two independent $\mathrm{T}_{3}$ AtPAP9 OE lines (P9OE5 and P9OE10) are shown. (B) Immunoblot analysis of total leaf protein using anti-AtPAP2 and anti-AtPAP9 antibodies, and gel staining of rubisco as the loading control. (C,D) Chlorophyll fluorescence analysis. Light-intensity-dependent NPQ (C) and ETR (D) were plotted. Data for each line are presented as the mean \pm SE ( $n>10$ per line). The statistical difference was determined by one-way ANOVA followed by multiple comparisons using the Tukey method. Asterisks indicate that the values of P2OE7 are significantly higher than those in the WT line $\left({ }^{*} p \leq 0.05\right)$. P9OE lines and pap9 did not exhibit any significant difference in both parameters compared to the WT line. The plants were dark-adapted for $1 \mathrm{~h}$ before measurement.

Under long-day $\left(16 \mathrm{~h}\right.$ light at $22^{\circ} \mathrm{C} / 8 \mathrm{~h}$ dark at $\left.18^{\circ} \mathrm{C}\right)$ growth conditions, the $\mathrm{OE}$ lines of AtPAP9 did not display any growth promotion effect compared with wild-type Arabidopsis thaliana ecotype Columbia-0 (WT) and T-DNA lines (Figure 2A). Overall, the typical early-bolting and high-seed-yield phenotypes of the AtPAP2 OE line (OE7) were not observed in the OE lines of AtPAP9 (Table 1) [8,19]. The electron transport rate (ETR) and non-photochemical quenching (NPQ) of the AtPAP9 OE and T-DNA lines were similar to the WT line, which correlated with their growth phenotypes (Figure 2C).

Table 1. Seed yield at maturity.

\begin{tabular}{ccc}
\hline Lines & Siliques per Plant & Seed Yield (g per Plant) \\
\hline WT & $367 \pm 50^{\mathrm{a}}$ & $0.203 \pm 0.047^{\mathrm{a}}$ \\
PAP9 & $353 \pm 34^{\mathrm{a}}$ & $0.198 \pm 0.062^{\mathrm{a}}$ \\
P2OE7 & $577 \pm 81^{\mathrm{b}}$ & $0.313 \pm 0.093^{\mathrm{b}}$ \\
P9OE5 & $350 \pm 36^{\mathrm{a}}$ & $0.209 \pm 0.077^{\mathrm{a}}$ \\
P9OE10 & $358 \pm 47^{\mathrm{a}}$ & $0.230 \pm 0.061^{\mathrm{a}}$ \\
\hline
\end{tabular}

$n=12$. Statistically significant difference was determined by one-way ANOVA followed by multiple comparisons using the Tukey method using GraphPad 7.04. Within each column, the values marked by different letters $(a, b)$ are significantly different $(p<0.05)$.

\subsection{AtPAP9 Modulates the Import Rate of $\left[{ }^{35} \mathrm{~S}\right]$-Labeled AtSSU1B into Chloroplasts}

AtPAP2 interacts with the mature protein portion of a small subunit of rubisco (mSSU) and a number of chloroplast proteins, and plays a role in the import of pSSU into chloroplasts [11,19]. To compare the binding specificities of AtPAP2 and AtPAP9, yeast twohybrid $(\mathrm{Y} 2 \mathrm{H})$ assay (Figure 3A) and bimolecular fluorescence complementation assay (BiFC) (Figure 3B) were carried out. The data of both assays confirmed that AtPAP9, like AtPAP2, can bind to the precursor of SSU (pSSU), mSSU, and a number of chloroplast proteins with the same specificity (Figure 4) [19]. Among the photosystem proteins, AtPAP9 interacted with the proteins located at the acceptor side of PSI, including Fd1, Fd2, root FNR1, root FNR2, FTRA2, FTRB, PsaE1, PsaE2, and three PsbQ-like proteins of the $\mathrm{NDH}$ subcomplexes (Figure 3A). In contrast, like AtPAP2, AtPAP9 did not interact with PsbQ1/Q2, PGRL1A/B, or the LHC proteins we tested. We also tested whether AtPAP9 can interact with Toc33 and Toc34, as all these proteins are anchored to the outer membranes of chloroplasts via their C-terminal transmembrane motifs and play a role in chloroplast protein import. Our data showed that they do not interact with each other (Figure 3). 

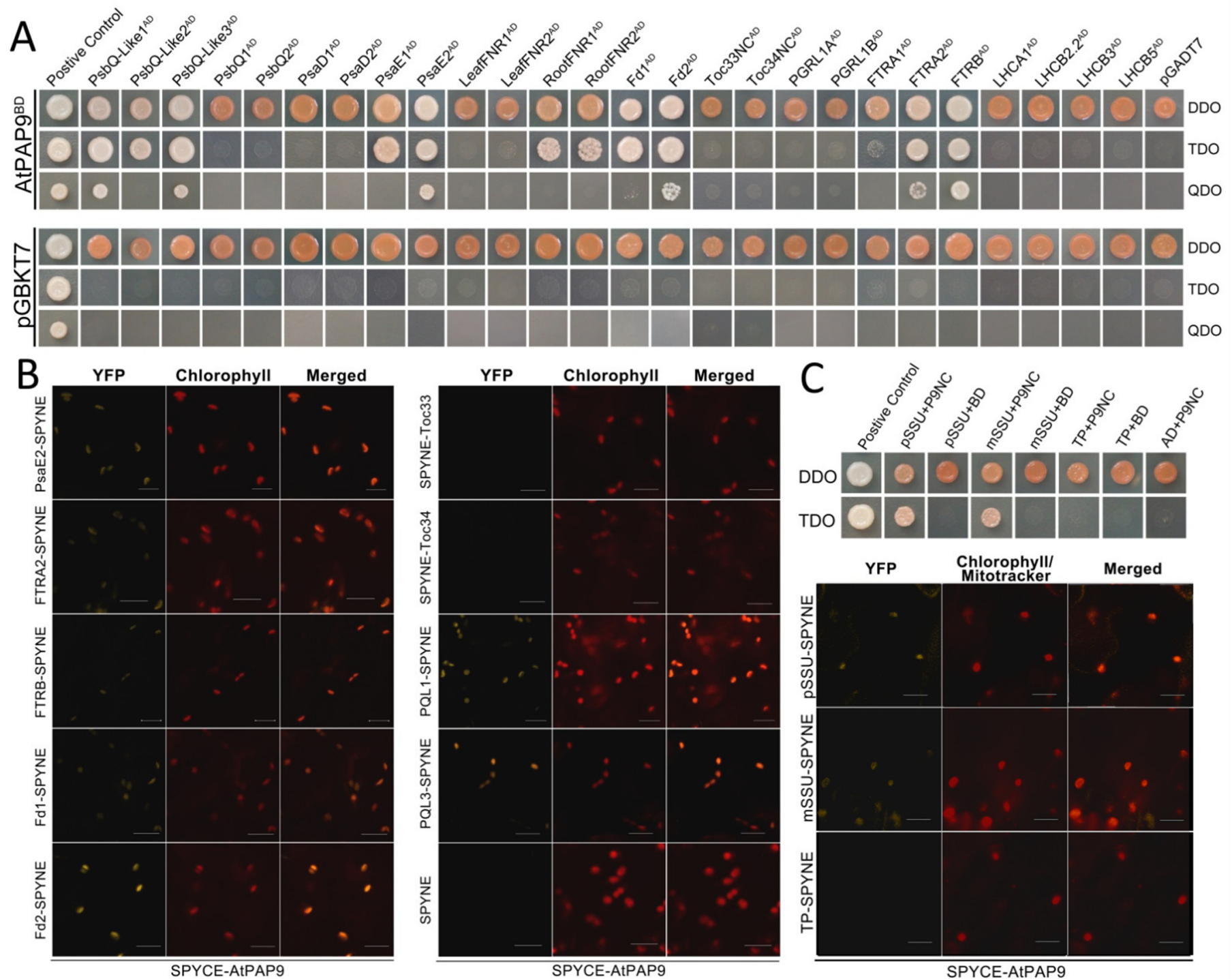

Figure 3. AtPAP9 interacted with a number of chloroplast proteins. (A) In Y2H assay, AtPAP9 without its signal peptide and C-terminus (a.a. 21-605, P9NC) was used as the bait and cloned into the pGBKT7 BD vector. It was then co-transformed with the pGADT7 AD vectors carrying the prey. To test for auto-activation, blank pGBKT7 BD vector and pGADT7 AD vector were co-transformed with the bait and prey, respectively. The yeast transformants were diluted 10x and selected on double-dropout (DDO, SD/-Leu/-Trp), triple-dropout (TDO, SD/-Leu/-Trp/-His), and quadruple-dropout (QDO, $\mathrm{SD} /$-Ade/-Leu/-Trp/-His) media. Yeast colonies grown on DDO medium indicated that both bait and prey vectors were successfully transformed into the cells, while yeast colonies grown on TDO and QDO media indicated medium and strong interaction of the bait and the prey in the yeast cells, respectively. pGBKT7-53 and pGADT7-T vectors were used as positive controls. (B) BiFC assay was carried out to confirm the interaction between AtPAP9 and the interacting proteins. YFPN-fused chloroplast proteins and YFPC-AtPAP9 were co-expressed in tobacco leaves by syringe injection. Empty SPYNE vectors were used as negative controls. Reconstituted YFP fluorescence was excited at $515 \mathrm{~nm}$ and detected with a PMT detector at an emission bandwidth of 530-550 nm (left panel). Chlorophyll autofluorescence was excited at $488 \mathrm{~nm}$ and detected with a PMT detector at an emission bandwidth of 650-710 nm (middle panel). An overlay of the YFP signal and the chlorophyll autofluorescence is shown in the right panel. Scale bars $=20 \mu \mathrm{m}$. All images were captured at the same gain settings as the corresponding PMT channels. (C) AtPAP9 interacted with pSSU (a.a. 1-181) and mSSU (a.a. 42-181) but not with the transit peptide of SSU (TP, a.a. 1-50 of pSSU) in $\mathrm{Y} 2 \mathrm{H}$ and BiFC assays. 
A

10\% WT pap9 OE10 WT pap9 OE10 WT pap9 OE10

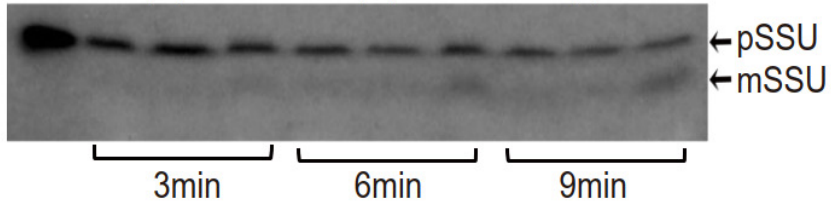

C
B

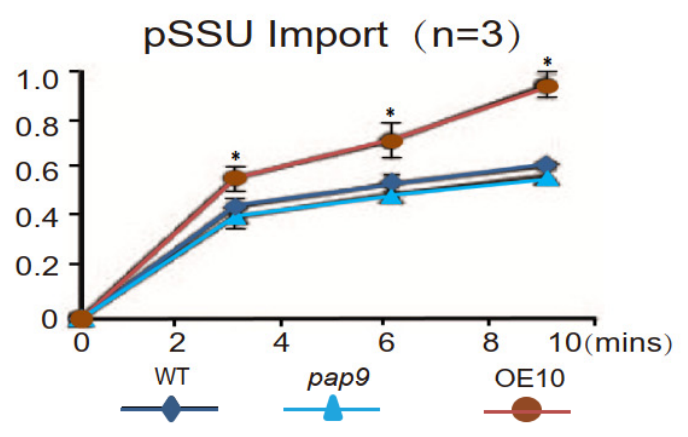

Figure 4. Import assay of $\left[{ }^{35} \mathrm{~S}\right]$-labeled pSSU into chloroplasts. (A) Chloroplast import reaction contained chloroplasts equivalent to $16 \mu \mathrm{g}$ of chlorophyll and $6 \mu \mathrm{L}$ of wheat germ extract (WGE)-synthesized pSSU; $10 \%$ of labeled pSSU added to the import assay was used as a loading control. (B) Qualification of import assay in (A) by image J. The maximal amount of imported $\mathrm{mSSU}$ in the OE10 line at $9 \mathrm{~min}$ was taken as 1.0. The import rate of pSSU into OE10 chloroplasts was significantly higher than that of WT and pap9 chloroplasts $\left({ }^{*} p \leq 0.05\right)$. Statistical difference was determined by one-way ANOVA followed by multiple comparisons using the Tukey method. (C) Chloroplasts isolated from WT, pap9, and OE10 lines were separated by $12 \%(v / v)$ SDS-PAGE, and Western blotting was carried out using anti-AtPAP2 and anti-AtPAP9 antibodies.

AtPAP2 on the outer membrane of chloroplasts modulates the import of $\left[{ }^{35} \mathrm{~S}\right]-\mathrm{pSSU}$ into chloroplasts [11]. Hence, we compared the import efficiency of [ $\left.{ }^{35} \mathrm{~S}\right]-p S S U$ into chloroplasts of the WT, pap9 (T-DNA), and AtPAP9 OE10 lines. The chloroplasts of the OE10 line imported $\left.{ }^{35} \mathrm{~S}\right]-\mathrm{pSSU}$ at a significantly higher rate than that of the WT line, while the import rate of $\left[{ }^{35} \mathrm{~S}\right]$-pSSU into pap 9 chloroplasts was similar to that into WT chloroplasts, possibly due to the presence of AtPAP2 on the pap 9 chloroplasts (Figure 4B). Hence, AtPAP9, like AtPAP2, also plays a role in pSSU import into chloroplasts.

\section{Discussion}

Brassica species tend to possess more than one copy of PAPs with a hydrophobic C-terminal motif, such as Camelina sativa (two copies) [24], Brassica rapa (four copies), and Brassica napus (eight copies). However, most other plant species usually only have one copy of PAP with a hydrophobic C-tail in their genomes, such as the smallest free-living photosynthetic eukaryote (Ostreococcus tauri) [8]. The presence of multiple copies of the AtPAP2-like gene reflects the ancient polyploidization events in the family Brassicaceae [25]. AtPAP9 is highly expressed in the roots, stems, and senescent leaves but is less expressed in photosynthetically active tissue, such as mature rosette leaves, cauline leaves, and developing siliques (Figure S3). This expression pattern is similar to that of AtPAP2 [8], and hence, they may have evolved by gene duplication in Brassicaceae during evolution.

AtPAP2 and AtPAP9 share a 72\% amino acid sequence identity (Figure S2). Four functional domains can be identified on the coding sequences of AtPAP2 and AtPAP9-an $\mathrm{N}$-terminal peptide, a fibronectin type III domain (FN-III-like), a phosphatase domain, and a hydrophobic motif at the C-termini (Figure 5A). Three-dimensional (3D) modeling based on the X-ray structure of Lupines luteus purple acid phosphatase PPD1 [26] predicted that AtPAP2 and AtPAP9 have highly similar 3D structures (Figure 5B,C). While the putative transit peptides of AtPAP2 and AtPAP9 are highly homologous (Figure S1), the putative transit peptide of AtPAP2 does not play a targeting function [8]. AtPAP2 is a 
tail-anchored protein dually targeted to chloroplasts and mitochondria via its C-terminal transmembrane motif $[8,9]$. GFP-targeting experiments demonstrated that the native Cterminal hydrophobic motif of AtPAP9 can only target GFP to chloroplasts (Figure 1). The amino acid differences in the C-tails of AtPAP2 and AtPAP9 after the gene duplication event in the Arabidopsis genome may have affected their ability to target to mitochondria. $\mathrm{Y} 2 \mathrm{H}$ and BiFC experiments indicated a similar protein-binding specificity between AtPAP9 and AtPAP2 (Figure 3) [19]. The chloroplast import assay using [ $\left.{ }^{35} \mathrm{~S}\right]-$ labeled AtSSU1B showed that OE of AtPAP9, like AtPAP2, can enhance the import of AtSSU1B into chloroplasts [11]. This is also supported by the 3D modeling of this enzyme in which a similar spatial arrangement between AtPAP2 and AtPAP9 was observed (Figure 5B).

OE of AtPAP2 on both chloroplasts and mitochondria simultaneously modulates their activities so that the plant cells can generate more ATP and sucrose, thus promoting plant growth [19]. While AtPAP9 shares a 72\% sequence identity with AtPAP2, no early-bolting and high-seed-yield phenotypes like those of the AtPAP2 OE7 line were observed in the OE lines of AtPAP9 (Figure 2). This raised the question of whether the function of AtPAP9's phosphatase domain is different from that of AtPAP2. Y2H assay indicated similar ligandbinding abilities of AtPAP2 and AtPAP9 to a number of chloroplast proteins (Figure 3A), but we cannot rule out the possibility that they differentially interact with some other substrates. Another possible reason for different growth phenotypes of the AtPAP2 and AtPAP9 OE lines (Figure 2) could be the inability of AtPAP9 to target to mitochondria. AtPAP2 plays a role in the import of multiple organellar RNA editing factor 3 (MORF3) into mitochondria [10]. Overexpression of AtPAP2 on mitochondria enhances its capacity to consume reducing equivalents, which could indirectly resupply $\mathrm{NADP}^{+}$in the stroma to accept electrons from the LEF [19]. As AtPAP9 is not targeted to the outer membrane of mitochondria, it is unlikely that its overexpression has any direct impact on mitochondria.

Another piece of evidence showing that AtPAP9 performs a different role from AtPAP2 is the growth phenotypes of their T-DNA lines. The T-DNA insertion site of atpap9 (Salk_129905) is located at the first exon (+465 nucleotides from the ATG start codon), and the line can grow like the WT line under normal growth conditions (Figure 2). However, a study reported that T-DNA insertion at the second exon of AtPAP9 exerts an embryonic lethal effect [21]. In contrast, the AtPAP2 T-DNA line (atpap2, Salk_013567), which also carries a T-DNA insertion at the second exon, does not exhibit an obvious growth difference compared with the WT line [8]. This further supports functional differences between AtPAP2 and AtPAP9. Collectively, AtPAP9 is functionally distinct from AtPAP2, although it may perform a redundant role in assisting the import of some preproteins into chloroplasts.

Interestingly, the overexpression of AtPAP2 in Arabidopsis, Camelina [27], and potato [28] promotes plant growth and yield, whereas the overexpression of AtPAP9 in Arabidopsis has no growth-promoting effects. This raises a question on the evolutionary relationship between AtPAP2 and AtPAP9. One possibility is that AtPAP2 is a gain-offunction of AtPAP9 during gene duplication; the other is that AtPAP9 evolved later and is a loss-of-function of its paralogous partner AtPAP2. Further functional comparison between these OE lines, including characterization of their chloroplasts and mitochondria, will lead to a better understanding of the physiology of these two energy-generating organelles. 


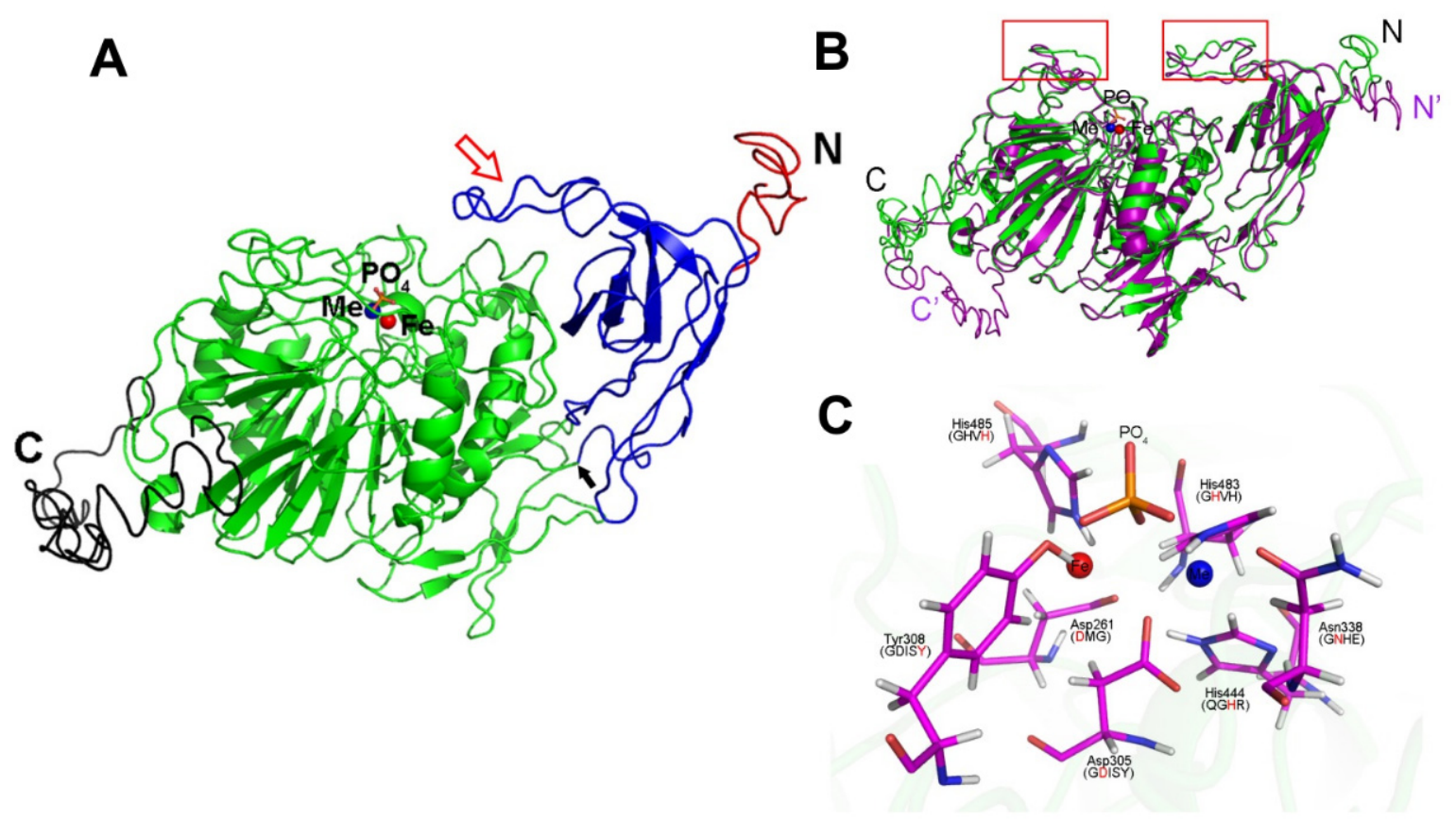

Figure 5. Predicted structure of AtPAP9. (A) Different domains of an AtPAP9 monomer unit are shown in different colors (red, transit peptide; blue, FN-III-like domain; green, PAP catalytic domain; black, C-terminal hydrophobic motif; blue sphere, $\mathrm{Me}\left(\mathrm{Zn}\right.$ or $\mathrm{Mn}$ ) atom; red sphere, Fe atom; orange spheres, $\mathrm{PO}_{4}$ ). The flexible loop is indicated by a red arrow, and the hinge connecting the FN-III-like and PAP catalytic domains is indicated by a black arrow. (B) The alignment of AtPAP2 (purple) and AtPAP9 (green) is shown as a cartoon by indicating their N- and C-termini, respectively. The metal atoms and phosphate ligands are displayed as spheres. The main different areas are boxed in red frames. (C) Active site and bound phosphate of AtPAP9. The Fe and Me atoms are displayed as red and blue spheres, respectively. The phosphate ligands are shown as sticks (red and yellow). The Fe atom is coordinated by amino residues His485, Tyr308, Asp261, and Asp305. The Me atom is coordinated by His444, His 483, Asn338, and Asp305. All the metal-chelating residues are shown as sticks as well by labeling the five conserved PAPs motifs (DMG, GDISY, GNHE, QGHR, and GHVH).

\section{Materials and Methods}

\subsection{Plant Materials and Growth Conditions}

The T-DNA insertion mutants of AtPAP9 (Salk_129905, Col-0) and AtPAP2 (Salk_129905, Col-0) were obtained from the Arabidopsis Biological Resource Center (ABRC, http: / /abrc.osu.edu/ (accessed on 1 July 2021)) and were verified as homozygous mutants by genomic PCR and RT-PCR [8]. Arabidopsis seeds were sterilized with $20 \%(v / v)$ bleach for $15 \mathrm{~min}$. The seeds were sown on Murashige and Skoog (MS) agar supplemented with $2 \%$ $(w / v)$ sucrose. To obtain soil-grown plants, 10-day-old seedlings were transferred to soil, where they were allowed to grow under long-day $\left(16 \mathrm{~h}\right.$ light at $22{ }^{\circ} \mathrm{C} / 8 \mathrm{~h}$ dark at $\left.18{ }^{\circ} \mathrm{C}\right)$ growth conditions.

\subsection{Isolation of Chloroplasts from Arabidopsis Leaves}

Arabidopsis seeds were sown and grown on Murashige and Skoog (MS) agar supplemented with $2 \%(w / v)$ sucrose under long-day $\left(16 \mathrm{~h}\right.$ light at $22{ }^{\circ} \mathrm{C} / 8 \mathrm{~h}$ dark at $\left.18^{\circ} \mathrm{C}\right)$ growth conditions. Chloroplasts were isolated from the leaves of 14-day-old Arabidopsis plants using the cellulase method $[29,30]$.

\subsection{Generation of Overexpression Lines of AtPAP9}

The full-length coding sequence (CDS) of AtPAP9 (At2g03450) cDNA was amplified with Pfx DNA polymerase (Invitrogen) using primers P9-F and P9-R (Table S2). The $1956 \mathrm{bp}$ PCR product was cloned into the plant transformation $\mathrm{pCXSN}$ vector via TA cloning [31]. The expression of AtPAP9 was driven by the CaMV 35S promoter (pCXSNCaMV35: AtPAP9). Plant transformation was performed, as previously described [32]. 
Seeds were screened on MS agar plates containing $30 \mu \mathrm{g} / \mathrm{mL}$ of hygromycin. $\mathrm{T}_{1}$ seedlings were transferred to soil and subjected to Western blotting analyses. $\mathrm{T}_{2}$ seedlings that followed a segregation pattern (resistant:susceptible $=3: 1$ ) were selected and transferred to soil. Homozygous $\mathrm{T}_{3}$ lines were subsequently identified for further analysis.

\subsection{Western Blotting Analysis}

The antibody of AtPAP9 was produced by PolyExpress ${ }^{\mathrm{TM}}$ Antibody Services (GenScript, Nanjing, China). The AtPAP9-specific peptide (a.a. 450-468, YTTSRKIRDAAIREKMIEH) designed using the OptimumAntigen design tool (GenScript, Nanjing, China) was used to immunize rabbits (Figure S2). The antibodies (anti-AtPAP9) were affinitypurified by the peptides. Anti-PAP2 antibodies were generated, as previously described [8] Horseradish peroxidase (HRP)-labeled secondary antibodies were used, and protein bands were visualized using the WesternBright ${ }^{\mathrm{TM}}$ Quantum Western blotting detecting kit (Advansta, San Jose, LA, USA).

\subsection{Subcellular Localization Analysis by GFP}

Arabidopsis mesophyll protoplast preparation and transfection were carried out, as previously described [33]. Briefly, $0.1 \mathrm{~mL}\left(10^{5}\right)$ of protoplasts isolated from 4-week-old rosettes were transfected with $8 \mu \mathrm{g}$ of plasmids and cultured for $16 \mathrm{~h}$ for protein expression. Plasmids for transient expression were extracted using a Hipure EF Plasmid Kit (Magen, Shenzhen, China). The C-terminal motif of AtPAP9 (a.a. 606-651) was amplified with the primer pairs P9C2GFP-F and P9CGFP-R (Table S2) and cloned into a GFP vector (pBI221) to generate the plasmid GFP-P9C. GFP-P2C1 (a.a. 615-656 of AtPAP2) was used as a control for comparison [9]. Chlorophyll and mitochondrial F1-RFP were used as organelle markers [34].

\subsection{Yeast Two-Hybrid Assay and Bimolecular Fluorescence Complementation Analysis}

The Matchmaker ${ }^{\mathrm{TM}}$ Gold Yeast Two-Hybrid system was used (Tanaka, Kasatsu, Japan). The PCR product of AtPAP9 (a.a. 21-605), lacking its N-terminal peptide and C-terminal transmembrane motif, was cloned to the C-terminus of the GAL4 DNA-binding domain (BD) of the pGBKT7 vector. The pGADT7 vectors carrying potential interacting partners were prepared, as previously described [11,19]. pGBKT7-53 and pGADT7-T vectors were used as positive controls. 3-AT was not used in the $\mathrm{Y} 2 \mathrm{H}$ assay. For BiFC assay, the fulllength cDNA of AtPAP9 was cloned into the PSPYNE vector, while the PSPYCE vectors carrying interacting partners were prepared, as previously described [11,19]. $\mathrm{Y} 2 \mathrm{H}$ and BiFC assays were carried out, as previously described [35].

\subsection{Chloroplast Import Assays}

Import-competent chloroplasts were isolated from 14-day-old Arabidopsis seedlings growing on MS agar plates, as previously described [29]. The [ $\left.{ }^{35} \mathrm{~S}\right]-$ labeled AtSSU1B (At5g38430) precursor protein was translated in vitro using the $\mathrm{TNT}^{\circledR}$-Coupled Wheat Germ Extract (WGE) System (Promega Corporation) [11]. The post-ribosome supernatant was prepared from freshly translated $\left[{ }^{35} \mathrm{~S}\right]$-AtSSU1B in WGE $\left(48,100 \times g, 20 \mathrm{~min}, 4{ }^{\circ} \mathrm{C}\right)$. The chloroplast import reaction was performed under white light at $25^{\circ} \mathrm{C}$ in import buffer containing $50 \mathrm{mM} \mathrm{HEPES} / \mathrm{KOH}, 3 \mathrm{mM} \mathrm{MgSO}_{4}, 0.33 \mathrm{M}$ sorbitol, $20 \mathrm{mM} \mathrm{K}$-gluconate, $10 \mathrm{mM}$ $\mathrm{NaHCO}_{3}, 0.2 \%(w / v)$ BSA, $5 \mathrm{mM} \mathrm{Mg}$-ATP, and $10 \mathrm{mM}$ methionine at $\mathrm{pH} 8.0[11,36]$. The import reaction was terminated with an equal volume of stop buffer $(50 \mathrm{mM} \mathrm{HEPES} / \mathrm{KOH}$, $0.33 \mathrm{M}$ sorbitol, and $50 \mathrm{mM}$ EDTA).

\subsection{Chlorophyll Fluorescence Measurements}

The chlorophyll fluorescence levels of Arabidopsis leaves were monitored with IMAGING-PAM M-Series Maxi Version (WALZ, Effeltrich, Germany). The plants were dark-adapted for at least $1 \mathrm{~h}$ before measurement. After the maximum $\left(\mathrm{F}_{\mathrm{m}}\right)$ and initial $\left(\mathrm{F}_{\mathrm{o}}\right)$ fluorescence values were determined with a delay of $40 \mathrm{~s}$, the plants were illuminated at 
the following light intensities: $0,81,145,186,281,335,461,701$, and $926 \mu \mathrm{mol}$ photon $\mathrm{m}^{-2}$ $\mathrm{s}^{-1}$. The duration of illumination at individual light intensities was $3 \mathrm{~min}$. A saturation pulse was applied at the end of each 3 min illumination.

\subsection{D Computer Modeling}

The structure of 3ZK4 (purple acid phosphatase PPD1 isolated from Lupines luteus) was previously found to be the best-fitting structure of AtPAP2 and AtPAP9 in the NCBI (https:/ /blast.ncbi.nlm.nih.gov (accessed on 1 July 2021)). Therefore, 3ZK4 was used as a reference to align the predicted structure of AtPAP2 and AtPAP9 with I-TASSER (http:/ / zhanglab.ccmb.med.umich.edu/I-TASSER/ (accessed on 1 July 2021)). PyMOL v1.3r1 (released in 2010) was used for subsequent editing.

Supplementary Materials: The following are available online at https:/ /www.mdpi.com/article/10 $.3390 /$ ijms $22147243 /$ s1.

Author Contributions: B.L.L. designed the study and wrote the manuscript. R.Z. carried out chloroplast import experiments, produced transgenic plants, and wrote the manuscript. M.Y. carried out protoplast transformation. X.G., Y.-S.L. and J.Y. performed Y2H and BiFC. X.G. produced 3D models. C.P.V. carried out Maxi-PAM measurements. F.S. generated the homologous T-DNA line of AtPAP9. All authors have read and agreed to the published version of the manuscript.

Funding: This project was funded by the Hong Kong Research Grants Council Area of Excellence Scheme (AoE/M-403/16) and the Innovation and Technology Fund (Funding Support to State Key Laboratory of Agrobiotechnology) of the Hong Kong Special Administrative Region, China. Any opinions, findings, conclusions, or recommendations expressed in this publication do not reflect the views of the Government of the Hong Kong Special Administrative Region or the Innovation and Technology Commission.

Institutional Review Board Statement: Not applicable.

Informed Consent Statement: Not applicable.

Data Availability Statement: The data supporting the findings of this study are available within the article and its Supplementary Materials.

Acknowledgments: We thank James Whelan, Simon Law, and Chris Carrie for helpful discussions and feedback.

Conflicts of Interest: The authors declare no conflict of interest.

\section{References}

1. Li, D.P.; Zhu, H.F.; Liu, K.F.; Liu, X.; Leggewie, G.; Udvardi, M.; Wang, D.W. Purple acid phosphatases of Arabidopsis thalianaComparative analysis and differential regulation by phosphate deprivation. J. Biol. Chem. 2002, 277, 27772-27781. [CrossRef]

2. Schenk, G.; Guddat, L.T.; Ge, Y.; Carrington, L.E.; Hume, D.A.; Hamilton, S.; de Jersey, J. Identification of mammalian-like purple acid phosphatases in a wide range of plants. Gene 2000, 250, 117-125. [CrossRef]

3. Lung, S.C.; Leung, A.; Kuang, R.; Wang, Y.; Leung, P.; Lim, B.L. Phytase activity in tobacco (Nicotiana tabacum) root exudates is exhibited by a purple acid phosphatase. Phytochemistry 2008, 69, 365-373. [CrossRef]

4. Kuang, R.; Chan, K.H.; Yeung, E.; Lim, B.L. Molecular and biochemical characterization of AtPAP15, a purple acid phosphatase with phytase activity, in Arabidopsis. Plant Physiol. 2009, 151, 199-209. [CrossRef] [PubMed]

5. Kaida, R.; Satoh, Y.; Bulone, V.; Yamada, Y.; Kaku, T.; Hayashi, T.; Kaneko, T.S. Activation of beta-glucan synthases by wall-bound purple acid phosphatase in tobacco cells. Plant Physiol. 2009, 150, 1822-1830. [CrossRef]

6. Liang, C.Y.; Tian, J.; Lam, H.M.; Lim, B.L.; Yan, X.L.; Liao, H. Biochemical and molecular characterization of PvPAP3, a novel purple acid phosphatase isolated from common bean enhancing extracellular ATP utilization. Plant Physiol. 2010, 152, 854-865. [CrossRef] [PubMed]

7. Liao, H.; Wong, F.-L.; Phang, T.-H.; Cheung, M.-Y.; Francisca Li, W.-Y.; Shao, G.; Yan, X.; Lam, H.-M. GmPAP3, a novel purple acid phosphatase-like gene in soybean induced by $\mathrm{NaCl}$ stress but not phosphorus deficiency. Gene 2003, 318, 103-111. [CrossRef]

8. Sun, F.; Suen, P.K.; Zhang, Y.; Liang, C.; Carrie, C.; Whelan, J.; Ward, J.L.; Hawkins, N.D.; Jiang, L.; Lim, B.L. A dual-targeted purple acid phosphatase in Arabidopsis thaliana moderates carbon metabolism and its overexpression leads to faster plant growth and higher seed yield. New Phytol. 2012, 194, 206-219. [CrossRef] [PubMed]

9. Sun, F.; Carrie, C.; Law, S.; Murcha, M.W.; Zhang, R.; Law, Y.S.; Suen, P.K.; Whelan, J.; Lim, B.L. AtPAP2 is a tail-anchored protein in the outer membrane of chloroplasts and mitochondria. Plant Signal. Behav. 2012, 7, 927-932. [CrossRef] [PubMed] 
10. Law, Y.S.; Zhang, R.; Guan, X.; Cheng, S.; Sun, F.; Duncan, O.; Murcha, M.; Whelan, J.; Lim, B.L. Phosphorylation and dephosphorylation of the presequence of pMORF3 during import into mitochondria from Arabidopsis thaliana. Plant Physiol. 2015, 169, 1-12. [CrossRef]

11. Zhang, R.; Guan, X.; Law, Y.S.; Sun, F.; Chen, S.; Wong, K.B.; Lim, B.L. AtPAP2 modulates the import of the small subunit of Rubisco into chloroplasts. Plant Signal. Behav. 2016, 11, e1239687. [CrossRef] [PubMed]

12. Ghifari, A.S.; Gill-Hille, M.; Murcha, M.W. Plant mitochondrial protein import: The ins and outs. Biochem. J. 2018, 475, 2191-2208. [CrossRef] [PubMed]

13. Shi, L.X.; Theg, S.M. The chloroplast protein import system: From algae to trees. Biochim. Biophys. Acta 2013, $1833,314-331$. [CrossRef] [PubMed]

14. Lamberti, G.; Gugel, I.L.; Meurer, J.; Soll, J.; Schwenkert, S. The cytosolic kinases STY8, STY17, and STY46 are involved in chloroplast differentiation in Arabidopsis. Plant Physiol. 2011, 157, 70-85. [CrossRef] [PubMed]

15. Law, Y.S.; Ngan, L.; Yan, J.R.; Kwok, L.Y.; Sun, Y.Z.; Cheng, S.F.; Schwenkert, S.; Lim, B.L. Multiple kinases can phosphorylate the N-terminal sequences of mitochondrial proteins in Arabidopsis thaliana. Front. Plant Sci. 2018, 9, 982. [CrossRef] [PubMed]

16. Waegemann, K.; Soll, J. Phosphorylation of the transit sequence of chloroplast precursor proteins. J. Biol. Chem. 1996, 271, 6545-6554. [CrossRef]

17. Nickel, C.; Soll, J.; Schwenkert, S. Phosphomimicking within the transit peptide of pHCF136 leads to reduced photosystem II accumulation in vivo. FEBS Lett. 2015, 589, 1301-1307. [CrossRef] [PubMed]

18. Liang, C.; Zhang, Y.; Cheng, S.; Osorio, S.; Sun, Y.; Fernie, A.R.; Cheung, C.Y.M.; Lim, B.L. Impacts of high ATP supply from chloroplasts and mitochondria on the leaf metabolism of Arabidopsis thaliana. Front. Plant Sci. 2015, 6, 922. [CrossRef]

19. Voon, C.P.; Law, Y.S.; Guan, X.; Lim, S.L.; Xu, Z.; Chu, W.T.; Zhang, R.; Sun, F.; Labs, M.; Leister, D.; et al. Modulating the activities of chloroplasts and mitochondria promotes ATP production and plant growth. Quant. Plant Biol. 2021, 2, e7. [CrossRef]

20. Xu, Z.; Zhang, R.; Yang, M.; Law, Y.-S.; Sun, F.; Hon, N.L.; Ngai, S.M.; Lim, B.L. A balance between the activities of chloroplasts and mitochondria is crucial for optimal plant growth. Antioxidants 2021, 10, 935. [CrossRef]

21. Zamani, K.; Lohrasebi, T.; Sabet, M.S.; Malboobi, M.A.; Mousavi, A. Expression pattern and subcellular localization of Arabidopsis purple acid phosphatase AtPAP9. Gene Expr. Patterns 2014, 14, 9-18. [CrossRef] [PubMed]

22. Kleffmann, T.; Russenberger, D.; von Zychlinski, A.; Christopher, W.; Sjolander, K.; Gruissem, W.; Baginsky, S. The Arabidopsis thaliana chloroplast proteome reveals pathway abundance and novel protein functions. Curr. Biol. 2004, 14, 354-362. [CrossRef]

23. Dhanoa, P.K.; Richardson, L.G.; Smith, M.D.; Gidda, S.K.; Henderson, M.P.; Andrews, D.W.; Mullen, R.T. Distinct pathways mediate the sorting of tail-anchored proteins to the plastid outer envelope. PLoS ONE 2010, 5, e10098. [CrossRef]

24. Liang, C.; Liu, X.; Yiu, S.M.; Lim, B.L. De novo assembly and characterization of Camelina sativa transcriptome by paired-end sequencing. BMC Genom. 2013, 14, 146. [CrossRef] [PubMed]

25. Cheng, S.; van den Bergh, E.; Zeng, P.; Zhong, X.; Xu, J.; Liu, X.; Hofberger, J.; de Bruijn, S.; Bhide, A.S.; Kuelahoglu, C.; et al. The Tarenaya hassleriana genome provides insight into reproductive trait and genome evolution of crucifers. Plant Cell 2013, 25, 2813-2830. [CrossRef] [PubMed]

26. Antonyuk, S.V.; Olczak, M.; Olczak, T.; Ciuraszkiewicz, J.; Strange, R.W. The structure of a purple acid phosphatase involved in plant growth and pathogen defence exhibits a novel immunoglobulin-like fold. IUCr] 2014, 1, 101-109. [CrossRef] [PubMed]

27. Zhang, Y.; Yu, L.; Yung, K.F.; Leung, D.Y.; Sun, F.; Lim, B.L. Over-expression of AtPAP2 in Camelina sativa leads to faster plant growth and higher seed yield. Biotechnol. Biofuels 2012, 5, 19. [CrossRef] [PubMed]

28. Zhang, Y.; Sun, F.; Fettke, J.; Schottler, M.A.; Ramsden, L.; Fernie, A.R.; Lim, B.L. Heterologous expression of AtPAP2 in transgenic potato influences carbon metabolism and tuber development. FEBS Lett. 2014, 588, 3726-3731. [CrossRef] [PubMed]

29. Smith, M.D.; Schnell, D.J.; Fitzpatrick, L.; Keegstra, K. In vitro analysis of chloroplast protein import. Curr. Protoc. Cell Biol. 2003, 11.1-11.16. [CrossRef]

30. Lister, R.; Carrie, C.; Duncan, O.; Ho, L.H.; Howell, K.A.; Murcha, M.W.; Whelan, J. Functional definition of outer membrane proteins involved in preprotein import into mitochondria. Plant Cell 2007, 19, 3739-3759. [CrossRef]

31. Chen, S.; Songkumarn, P.; Liu, J.; Wang, G.L. A versatile zero background T-vector system for gene cloning and functional genomics. Plant Physiol. 2009, 150, 1111-1121. [CrossRef] [PubMed]

32. Clough, S.J.; Bent, A.F. Floral dip: A simplified method for Agrobacterium-mediated transformation of Arabidopsis thaliana. Plant J. 1998, 16, 735-743. [CrossRef] [PubMed]

33. Yoo, S.D.; Cho, Y.H.; Sheen, J. Arabidopsis mesophyll protoplasts: A versatile cell system for transient gene expression analysis Nat. Protoc. 2007, 2, 1565-1572. [CrossRef] [PubMed]

34. Carrie, C.; Kuhn, K.; Murcha, M.W.; Duncan, O.; Small, I.D.; O’Toole, N.; Whelan, J. Approaches to defining dual-targeted proteins in Arabidopsis. Plant J. 2009, 57, 1128-1139. [CrossRef]

35. Schweiger, R.; Schwenkert, S. Protein-protein interactions visualized by bimolecular fluorescence complementation in tobacco protoplasts and leaves. J. Vis. Exp. 2014, 85, e51327. [CrossRef] [PubMed]

36. Fitzpatrick, L.M.; Keegstra, K. A method for isolating a high yield of Arabidopsis chloroplasts capable of efficient import of precursor proteins. Plant J. 2001, 27, 59-65. [CrossRef] 